DOI 10.17816/transsyst201843s1124-133

(C) F. Costa, R. de Andrade, R. Stephan

Laboratory of Applied Superconductivity

Federal University of Rio de Janeiro

(Rio de Janeiro, Brazil)

\title{
CHARACTERIZATION OF LEVITATION FORCE FOR A SUPERCONDUCTING MAGNETIC LEVITATION VEHICLE
}

Background: In Superconducting Magnetic Levitation (SML) transportation systems, such as the MagLev-Cobra prototype, the levitation force plays an important role, both for efficiency and safety reasons.

Aim: Determination of the maximum load on the magnetic suspension.

Methods: To determine how much load the magnetic suspension system supports, numerical simulations, based on computational models, and laboratory experimental tests are normally used.

The most commonly way for characterization of a SML bearing is the measurement of the levitation force as a function of distance between a Superconductor (YBCO) $[1,2]$ and a Permanent Magnet Guideway (PMG). The measurement of levitation as a function of distance, the banana curve, has a hysteretic behavior with the results depending of the history of measurement: whether the distance between the superconductor and the PMG is decreasing the force is higher than when the distance is increasing, the force is higher with faster movements and so on. A different approach of levitation force test will be proposed as an alternative to the hysteresis curve. This method, applied to characterize the levitation dynamics of the MaglevCobra vehicle, provided more reliable and consistent data with the levitation dynamics observed during the operation of the real scale prototype in the transportation of passengers.

The bench-top levitation test emulates the behavior of the vehicle along its operation, regardless of the position history between the magnets and the superconductor materials. The test consists in placing the superconductor, refrigerated inside cryostats, in a predetermined Field Cooling (FC) position, and slowly move the cryostat above the PMG to a lower high, for example $15 \mathrm{~mm}$, and wait 10 minutes. After that, the high is decreased $1 \mathrm{~mm}$ and again hold there for 10 minutes. The procedure is repeated until a high of $10 \mathrm{~mm}$ is reached. The hole process is repeated at least 5 times. This routine replicates the load condition during the vehicle operation when passengers board the train and the load stay constant until the end of the journey. After that, another group of passengers takes place inside the vehicle and is carried to the next station.

This kind of test shows the creep of the levitation force over time with slow dynamics and gives the average load over height of levitation along the given time of operation, helping engineers to predict the load capacity of the vehicle and design a more reliable layout.

Results: Three FC positions were investigated. The position currently used by the MagLev-Cobra $(35 \mathrm{~mm}$ ) and other 2 positions $(45 \mathrm{~mm}$ and $55 \mathrm{~mm}$ ) of initial height between the superconductor and the permanent magnet guideway. 
Conclusion: All these results contribute to an improvement in the criticality index and a safe application of this system on transportation of persons.

Keywords: Superconducting Magnetic Levitation, Urban Transportation, Superconductors, Rare Earth Permanent Magnets, Force Measurements.

\section{INTRODUCTION}

In handling systems that use superconducting levitation [3], it is extremely important to make the most of the levitation force for both efficiency and safety reasons. To determine how much load the magnetic suspension system supports, it is possible to use models and numerical simulations or physical tests in the laboratory. Just as important as using a suitable mathematical model is to perform tests in a manner consistent with levitation and operation.

To determine the ideal levitation height of the Maglev, some bench tests were performed in the laboratory, in which the actual operating conditions were reproduced. It was decided to perform tests because the modeling of the phenomenon of levitation still be a subject not totally dominated by researchers around the world. Several theories attempt to reproduce the experimental results mathematically without success. The superconductors used in the Maglev Cobra are type II.

\section{THE HYSTERESIS CURVE}

The most used curve to characterize a superconducting bearing is the banana curve that represents a force versus displacement graph between a superconductor and a magnet (Fig. 1).

The repulsive force between a magnet and a superconductor is a complicated mechanism to be analyzed. What can be said is that the force increases as the superconductor approaches the magnet and decreases as it moves away. However, it is different when the two are moving away or approaching. When the magnet moves away, the repulsive force between the magnet and its image diminishes. In addition, flux pinning lines cause an attractive force that reduces liquid repulsive force. This results in a curve force vs distance with hysteresis.

Moreover, the equilibrium position depends on the amplitude of the excursion that the superconductor performed on the magnetic field of the magnet. As can be seen in figure1 there are 4 Hysteresis curves measured for the same magnet and superconductor assembly. The first curve with the largest displacement is the displacement curve of 25 to $10 \mathrm{~mm}$ where it is possible to observe the largest Hysteresis. The three following curves (2,3 and 4) are displacements (20 to $15 \mathrm{~mm}$ ), 


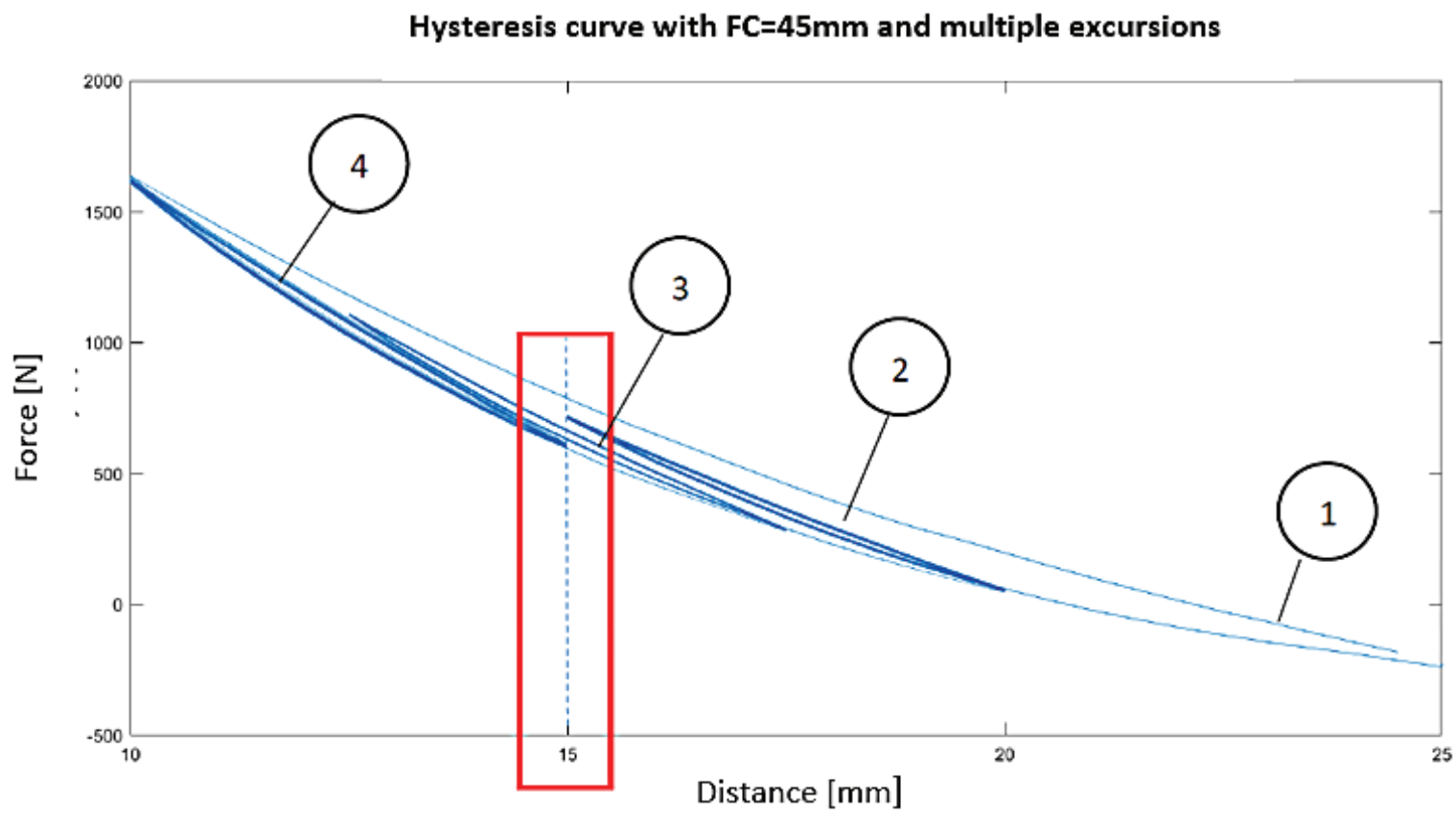

Fig. 1. The classical Banana curve for different amplitude excursions

(17.5 to $12.5 \mathrm{~mm}$ ) and (15 to $10 \mathrm{~mm}$ ), so it is impossible to determine the ordered pair (position, force) without knowing the displacement history of the assembly.

One can take as an example the position of $15 \mathrm{~mm}$ in the different curves. To know the force associated with this position it is necessary to know what displacement was done around this position.

\section{EXPERIMENTAL SETUP}

The levitation force testing system is illustrated in Fig. 2 and allows movements in the vertical direction. The equipment is connected to a computer that uses the Labview software both for control of the movement and for the acquisition of the data of the load cell. Other researchers also perform levitation tests in their laboratories $[4,5,6]$.

The test configurations are listed in Table 1.

Table 1.Test Configuration

\begin{tabular}{|c|c|}
\hline Step & $0.5 \mathrm{~mm}$ \\
\hline Speed & $3 \mathrm{~mm} / \mathrm{s}$ \\
\hline Sample rate & $10 \mathrm{kHz}$ \\
\hline Resolution & $3 / 4 \mathrm{~N}$ \\
\hline Waiting time after movement & $10 \mathrm{~min}$ \\
\hline
\end{tabular}




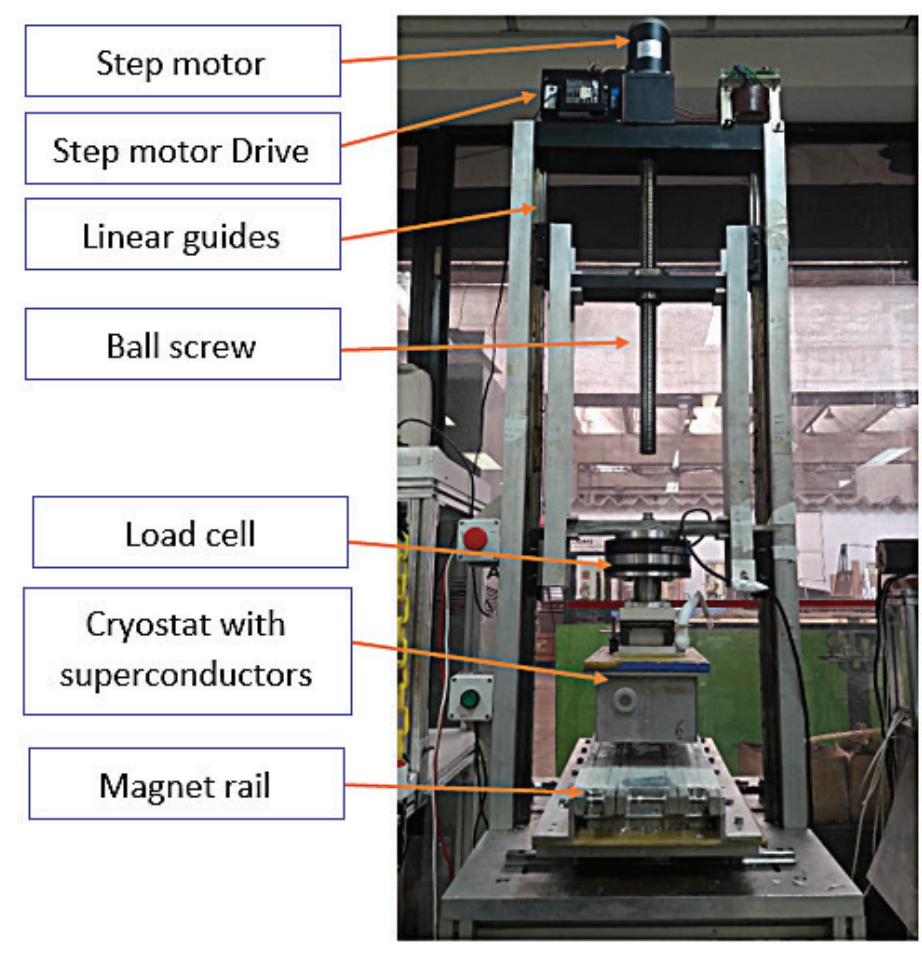

Fig. 2. Experimental Setup

\section{RESULTS AND DISCUTION}

In order to perform bench-top levitation tests, we tried to get closer to the behavior of the vehicle over the operating time [7].

Three Field Cooling positions were investigated (YBCO cooling in the presence of magnetic field). Position currently used by the Maglev $(35 \mathrm{~mm})$ and other 2 positions ( $45 \mathrm{~mm}$ and $55 \mathrm{~mm}$ ) of initial height between the superconductor and the rail. To evaluate the force as a function of the levitation gap throughout the day the procedure used was placing the superconductor, refrigerated inside cryostats, in a predetermined Field Cooling (FC) position, and slowly move the cryostat above the PMG to a lower high, for example $15 \mathrm{~mm}$, and wait 10 minutes. After that, the high is decreased $1 \mathrm{~mm}$ and again hold there for 10 minutes. The procedure is repeated until a high of $10 \mathrm{~mm}$ is reached. The hole process is repeated at least 5 times.

With this procedure, gradual loading of the cryostat is simulated throughout the day in the same way as the vehicle with the entry and exit of passengers at each trip. It is impossible, however, to repeat the exact loading that the vehicle suffers with the transport of passengers during its working period, as the load distribution of the vehicle is not homogeneous, and passengers will not always position themselves in the same place inside the vehicle. 
With the proposed test methodology, it is possible to evaluate the force curve by displacement over time in a discrete way by averaging each 10 -minute period evaluated and plotting the curve of these points for each position $(10,11,12,13$, 14 and $15 \mathrm{~mm}$ ).

The first test cycle represents the so-called field pumping when the superconductor is pressed for the first time against the magnetic field of the magnet, this action causes a stress in the pinning network and has the consequence called the Flux Creep and, therefore, decay of levitation force. This Creep of the first cycle is more pronounced than in the other cycles of tests and can be observed in the following three graphs that represent the first test cycle for each height of FC. Another observation is that the levitation force is always greater during field pumping than during the other stages of testing.

This phenomenon can be observed in Fig. 3, 4 and 5.

To observe the levitation force over the hours, the mean force measured over the 10 minutes was used as a representation of the cryostat's capacity in this time window. By performing several test cycles with at least 1-hour interval between them it was possible to use each midpoint along the hours.

With these results it is possible to observe the decay of the levitation force for different $\mathrm{FC}$ heights and to know how much load the vehicle can carry along the operating time depending on the height of Field Cooling used. The first hour of testing is not part of the tables because it is when the creep flux shown in the

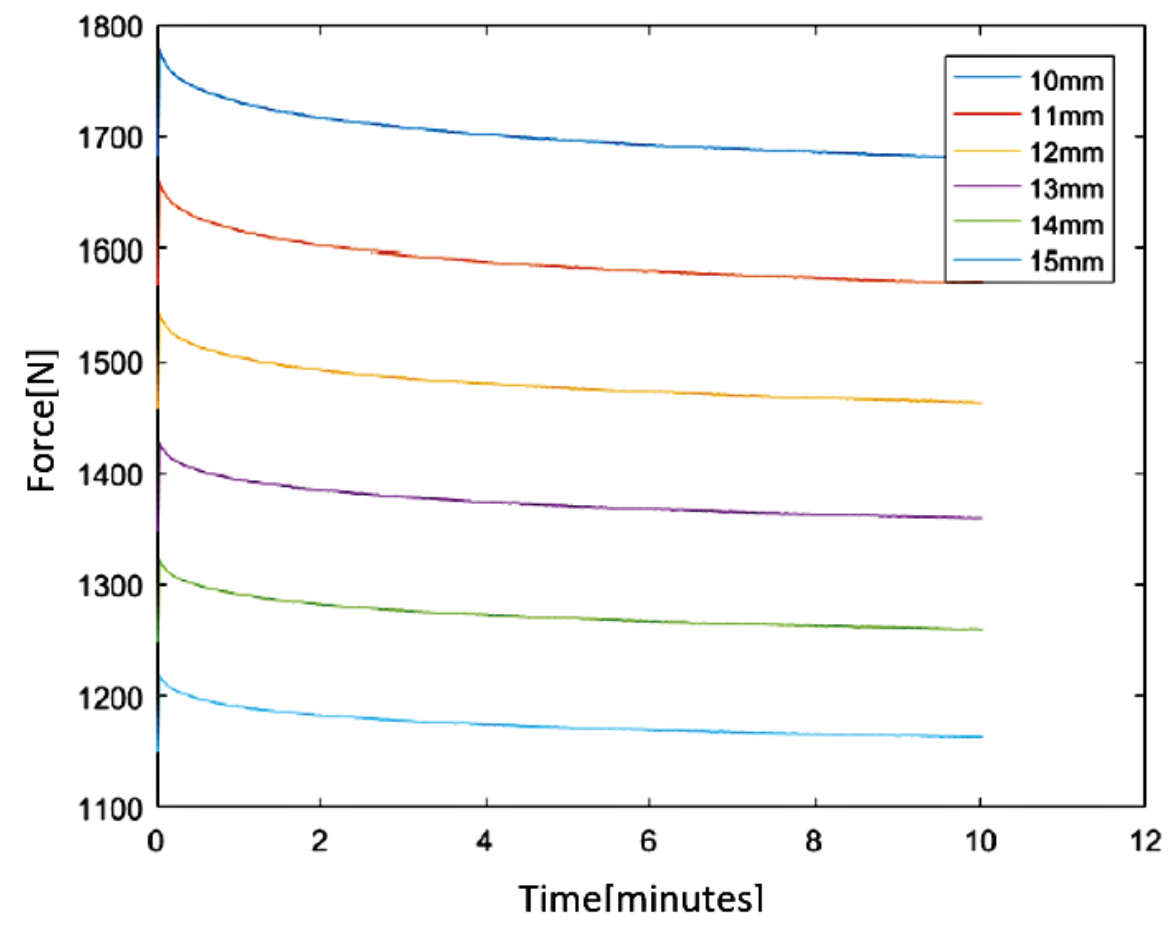

Fig. 3. First test (flux Creep) FC 35 mm 


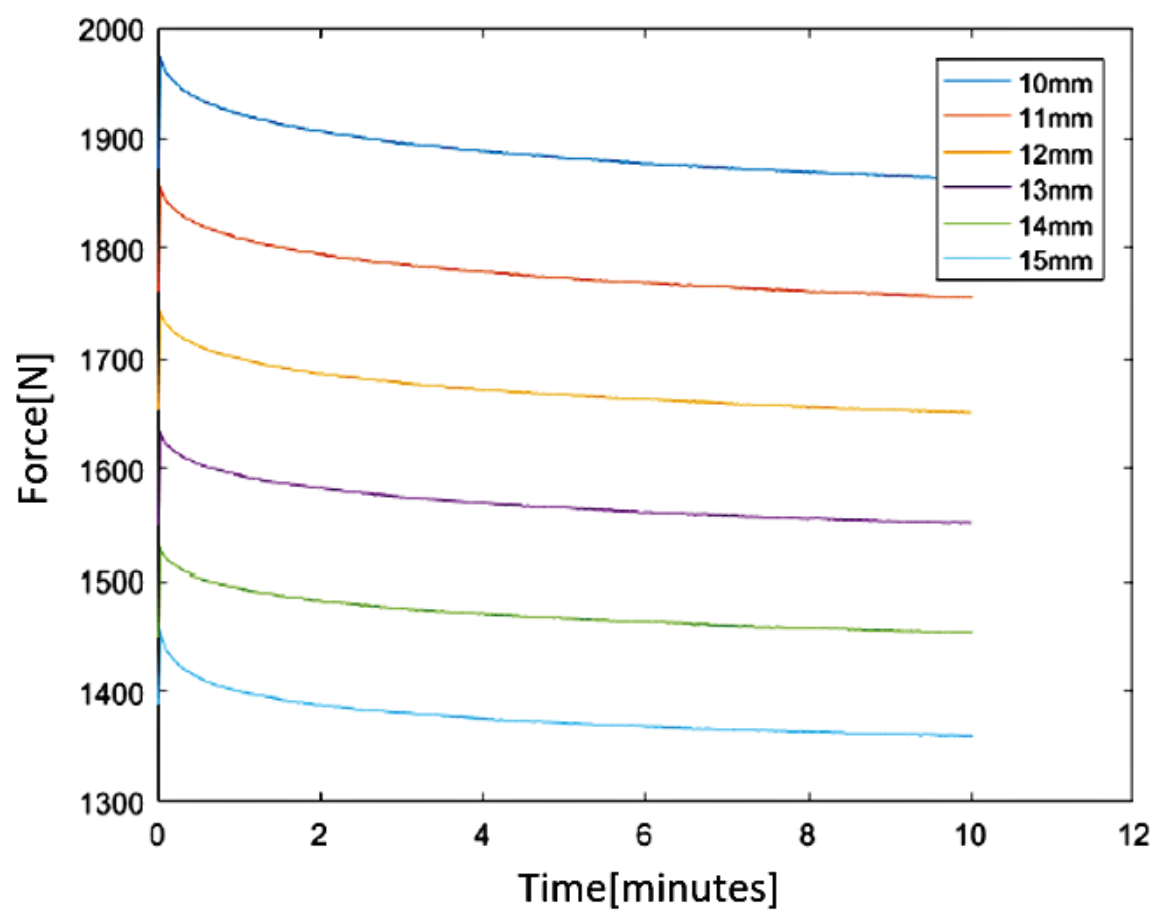

Fig. 4. First test (flux Creep) FC $45 \mathrm{~mm}$

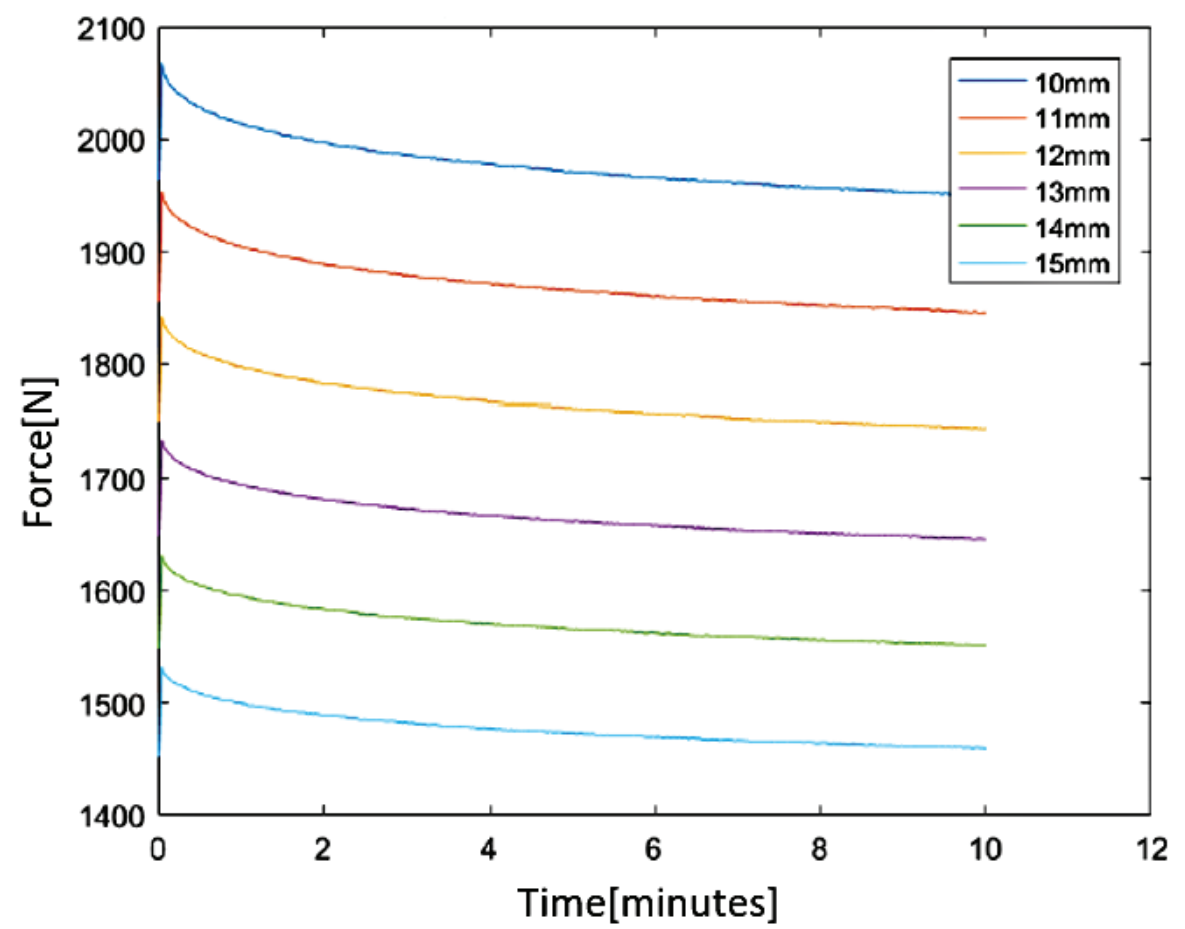

Fig. 5. First test (flux Creep) FC $55 \mathrm{~mm}$

previous Fig. 3, 4 and 5 happens and where the levitation force is larger but does not represent the true load that levitation is able to withstand. 
Table 2. Force Average by levitation Gap and accumulated losses (FC $35 \mathrm{~mm}$ )

\begin{tabular}{|c|c|c|c|c|c|}
\cline { 2 - 6 } \multicolumn{1}{c|}{} & \multicolumn{5}{c|}{ FC (35 mm) Force Average [N] } \\
\hline $\mathbf{m m}$ & Hour 2 & Hour 3 & Hour 4 & Hour 5 & Hour 6 \\
\hline 10 & 1667.90 & 1651.20 & 1641.52 & 1634.04 & 1628.09 \\
\hline $\mathbf{1 1}$ & 1484.18 & 1463.59 & 1450.55 & 1442.21 & 1435.34 \\
\hline $\mathbf{1 2}$ & 1297.11 & 1275.76 & 1262.84 & 1254.15 & 1247.79 \\
\hline $\mathbf{1 3}$ & 1117.60 & 1096.08 & 1083.46 & 1074.47 & 1068.38 \\
\hline $\mathbf{1 4}$ & 947.76 & 926.61 & 914.19 & 905.55 & 899.77 \\
\hline $\mathbf{1 5}$ & 787.78 & 767.73 & 755.41 & 745.88 & 740.21 \\
\hline
\end{tabular}

\begin{tabular}{|c|}
\hline $\begin{array}{c}\text { Accumulated } \\
\text { Losses }\end{array}$ \\
\hline $2.4 \%$ \\
\hline $3.3 \%$ \\
\hline $3.8 \%$ \\
\hline $4.4 \%$ \\
\hline $5.1 \%$ \\
\hline $6.0 \%$ \\
\hline
\end{tabular}

Table 3. Force Average by levitation Gap and accumulated losses (FC $45 \mathrm{~mm}$ )

\begin{tabular}{|c|c|c|c|c|c|}
\cline { 2 - 6 } \multicolumn{1}{c|}{} & \multicolumn{5}{c|}{ FC (45 mm) Force Average [N] } \\
\hline $\mathbf{m m}$ & Hour 2 & Hour 3 & Hour 4 & Hour 5 & Hour 6 \\
\hline 10 & 1851.23 & 1832.55 & 1821.05 & 1812.18 & 1805.15 \\
\hline 11 & 1665.48 & 1639.21 & 1624.55 & 1614.68 & 1607.53 \\
\hline $\mathbf{1 2}$ & 1470.76 & 1443.73 & 1429.11 & 1419.64 & 1413.06 \\
\hline $\mathbf{1 3}$ & 1284.01 & 1257.16 & 1242.73 & 1234.04 & 1227.79 \\
\hline $\mathbf{1 4}$ & 1105.37 & 1078.80 & 1064.85 & 1056.28 & 1050.79 \\
\hline $\mathbf{1 5}$ & 935.65 & 909.64 & 895.77 & 888.04 & 883.03 \\
\hline
\end{tabular}

\begin{tabular}{|c|}
\hline $\begin{array}{c}\text { Accumulated } \\
\text { Losses }\end{array}$ \\
\hline $2.5 \%$ \\
\hline $3.5 \%$ \\
\hline $3.9 \%$ \\
\hline $4.4 \%$ \\
\hline $4.9 \%$ \\
\hline $5.6 \%$ \\
\hline
\end{tabular}

Table 4. Force Average by levitation Gap and accumulated losses (FC $55 \mathrm{~mm}$ )

\begin{tabular}{|c|c|c|c|c|c|}
\cline { 2 - 6 } \multicolumn{1}{c|}{} & \multicolumn{5}{c|}{ FC (55 mm) Force Average [N] } \\
\hline $\mathbf{m m}$ & Hour 2 & Hour 3 & Hour 4 & Hour 5 & Hour 6 \\
\hline $\mathbf{1 0}$ & 1936.73 & 1915.56 & 1903.08 & 1893.23 & 1885.89 \\
\hline $\mathbf{1 1}$ & 1750.90 & 1721.99 & 1705.69 & 1693.83 & 1685.33 \\
\hline $\mathbf{1 2}$ & 1554.74 & 1524.63 & 1508.27 & 1496.01 & 1487.93 \\
\hline $\mathbf{1 3}$ & 1366.34 & 1336.08 & 1319.88 & 1307.74 & 1299.97 \\
\hline $\mathbf{1 4}$ & 1185.78 & 1155.57 & 1139.47 & 1127.74 & 1120.33 \\
\hline $\mathbf{1 5}$ & 1013.49 & 983.47 & 967.83 & 956.21 & 949.05 \\
\hline
\end{tabular}

\begin{tabular}{|c|}
\hline $\begin{array}{c}\text { Accumulated } \\
\text { Losses }\end{array}$ \\
\hline $2,6 \%$ \\
\hline $3,7 \%$ \\
\hline $4,3 \%$ \\
\hline $4,9 \%$ \\
\hline $5,5 \%$ \\
\hline $6,4 \%$ \\
\hline
\end{tabular}

As the vehicle weights $2300 \mathrm{kgf}(22540 \mathrm{~N})$, the number of passengers should be limited safely, since the number of cryostats is fixed at 24 units in this project.

Thus, the following scenarios are possible at the end of 6 hours of operation with $10 \mathrm{~mm}$ of levitation gap:

According to table 5, considering that each passenger weighs average of $80 \mathrm{kgf}$, it is possible to estimate the capacity of transport of the vehicle for each height of FC. 
Table 5. Overall Capacity by FC height

\begin{tabular}{|c|c|c|c|c|c|}
\hline $\begin{array}{c}\text { FC } \\
\text { height }\end{array}$ & $\begin{array}{c}\text { Capacity } \\
\text { by cryostat }\end{array}$ & $\begin{array}{c}\text { Total } \\
\text { capacity }\end{array}$ & $\begin{array}{c}\text { Total capacity } \\
\text { minus vehicle } \\
\text { weight }\end{array}$ & $\begin{array}{c}\text { Number of } \\
\text { passengers } \\
(80 \mathrm{~kg} \mathrm{f} / \text { person })\end{array}$ & $\begin{array}{c}\text { Safety index } \\
\text { with 15 } \\
\text { passengers }\end{array}$ \\
\hline $35 \mathrm{~mm}$ & $1628 \mathrm{~N}$ & $39072 \mathrm{~N}$ & $16532 \mathrm{~N}$ & 21 & 1,40 \\
\hline $45 \mathrm{~mm}$ & $1805 \mathrm{~N}$ & $43320 \mathrm{~N}$ & $20780 \mathrm{~N}$ & 26 & 1,76 \\
\hline $55 \mathrm{~mm}$ & $1885 \mathrm{~N}$ & $45240 \mathrm{~N}$ & $22700 \mathrm{~N}$ & 28 & 1,93 \\
\hline
\end{tabular}

The tests show that the force decay is small (2.4\% to $6.4 \%)$ for a period of eight hours after the Field cooling and that when the cryostat works more heavily and closer to the rail the loss of force levitation is lower.

Another result is that the quasi-static progressive loading used does not show a logarithmic decay as observed in dynamic Hysteresis type tests. Perhaps because in conducting the dynamic tests there is the movement of a superconductor in the magnetic field of the magnet, causing in addition to the creep, induction of currents inside the superconductor during the movement which in turn generates repulsive forces in the system increasing the levitation force.

With this kind of tests presented, it is possible to predict the behavior of the cryostat over the time of operation, it being enough to know which was the FC used and in which position the cryostats are of the rail. The latter information can easily be obtained by using distance sensors in the vehicle.

The graphs in Fig. 3, 4 and 5 represent the magnetic field pumping curves in the first test cycle explain why the Maglev Cobra levitates higher in the first trips causing the collision of the linear motor safety bearings, causing slight discomfort to the passengers.

\section{CONCLUSIONS}

These preliminary data suggest that it would be interesting to change the height of Field Cooling currently used in the Maglev Cobra from FC 35 to FC 45, thereby increasing the carrying capacity. For this change it would be necessary to also investigate the lateral force behavior that holds the vehicle in the path of the rail.

During the tests of levitation force, it was possible to observe that a curve of approximation of amplitude in the range of $5 \mathrm{~mm}$ no longer represented the actual load supported by the levitator, and to know the payload for each levitation height a static test should be used. With this, a new test methodology was adopted to approximate the maximum as possible the behavior of the vehicle during its 
operation. Also, was possible to observe that the levitation force for the cryostat is lower than expected for $10 \mathrm{~mm}$ distance, but the decay measured over time was lower than estimated according to the tables 2, 3 and 4 . These data should be confirmed when height sensors are fitted to the vehicle.

Through the tests the number of passengers inside the vehicle can be assessed with greater confidence and a safety coefficient can now be applied according to table 5. Thus, the failure mode - levitation loss due to overload can be mitigated. All these results contribute to an improvement in the criticality index and a safe application of this system on transportation of persons like proposed in $[8,9,10]$.

\section{ACKNOWLEDGMENT}

FAPERJ - Carlos Chagas Filho Foundation for Research Support of the State of Rio de Janeiro and CNPq - National Council for Scientific and Technological Development Brazil for the financial support.

\section{References}

1. Wu MK, Ashburn JR., Torng CJ, Hor PH, Meng RL, Gao L, Huang ZJ, Quang YQ, Chu CW. Superconductivity at $93 \mathrm{~K}$ in a New Mixed-Phase Y-Ba-Cu-O Compound System at Ambient Pressure. Physical Review Letters, 1987;58(9):908-910. doi: 10.1103/physrevlett.58.908

2. Murakami M., Oyama T, Fujimoto H, Taguchi T, Gotah S, Shiohara Y, Koshizuka N, Tamaka S. Large Levitation Force due to Flux Pinning in $\mathrm{YBaCuO}$ Superconductors Fabricated by Melt-Powder-Melt-Grouth Process. Japanese Journal of Applied Physic, 1990;29(2-11):1991-1994. doi: 10.1143/jjap.29.11991

3. Wang S, Wang J, Wang X, Ren Z, Zeng Y, Deng C, Jiang H, Zhu M, Lin G, Xu Z, Zhu D, Song H. The Man-Loading High-Temperature Superconducting MagLev Test Vehicle. IEEE Transactions on Applied Superconductivity, 2003;13(2):2134-2137. doi:10.1109/ tasc.2003.813017

4. Deng Z, Zhang W, Zheng J, Wang B, Ren Y, Zheng X, and Zhang J. A High-Temperature Superconducting Maglev-Evacuated Tube Transport (HTS Maglev-ETT) Test System. IEEE Transactions on Applied Superconductivity, 2017;27(6):1-8. doi: 10.1109/ tasc.2017.2716842

5. Deng Z, Zhang W, Zheng J, Ren Y, Jiang D, Zheng X, Zhang J, Gao P, Lin Q, Song B and Deng C. A High-Temperature Superconducting Maglev Ring Test Line Developed in Chengdu, China. IEEE Transactions on Applied Superconductivity, 2016;26(6):1-8. doi:10.1109/tasc.2016.2555921

6. Schultz L, de Haas O, Verges P, Beyer C, Rohlig S, Olsen H, Kuhn L, Berger D, Noteboom U, Funk U. Superconductively Levitated Transport System - the SupraTrans project, IEEE Transactions on Applied Superconductivity, 2005;15(2):2301-2305. doi:10.1109/ tasc. 2005.849636

7. Stephan RM, de Andrade Jr R, Santos G, Neves MA, Nicolsky R. Levitation Force and Stability of Superconducting Linear Bearings Using NdFeB and Ferrite Magnets. Physica. C, Superconductivity, 2003;386:490-494. doi: 10.1016/s0921-4534(02)02235-9 
8. Stephan RM, Nicolsky R, Neves MA, Ferreira AC, de Andrade Jr R, Moreira MAC, Rosário MAP, Machado OJ. A Superconducting Levitation Vehicle Prototype. Physica C, Superconductivity, 2004;408-410:932-934. doi:10.1016/j.physc.2004.03.169

9. Stephan RM, de Andrade Jr R., Ferreira AC. Superconducting Light Rail Vehicle: A Transportation Solution for Highly Populated Cities. IEEE Vehicular Technology Magazine, 2010;7(4):122-127. doi: 10.1109/mvt.2012.2218437

10. Stephan RM, de Andrade Jr. R, Ferreira AC, Sotelo GG. Superconducting Levitation Applied To Urban Transportation. Wiley Encyclopedia of Electrical and Electronics Engineering, 2017:1-18. doi: 10.1002/047134608X.W8346

Information about the authors:

Costa Felipe, PhD student;

ORCID: 0000-0002-9457-2805

E-mail: felipe@pee.ufrj.br

De Andrade Junior Rubens, DSc.;

ORCID: 0000-0003-3995-965X

E-mail: rubens.andrade.jr@poli.ufrj.br

Stephan Richard, Dr.-Ing.;

ORCID: 0000-0002-8019-1016

E-mail:rms@ufrj.br

\section{To cite this article:}

Costa F, De Andrade R, Stephan R. Characterization of Levitation Force for a Superconducting Magnetic Levitation Vehicle. Transportation Systems and Technology. 2018;4(3 suppl. 1):124133. doi: 10.17816/transsyst201843s1124-133 